\title{
Discrete vector on-site vortices
}

\author{
P.G. Kevrekidis ${ }^{1}$ and D.E. Pelinovsky ${ }^{2}$ \\ ${ }^{1}$ Department of Mathematics, University of Massachusetts, Amherst, Massachusetts, 01003-4515, USA \\ ${ }^{2}$ Department of Mathematics, McMaster University, Hamilton, Ontario, Canada, L8S 4K1
}

August 13, 2018

\begin{abstract}
We study discrete vortices in coupled discrete nonlinear Schrödinger equations. We focus on the vortex cross configuration that has been experimentally observed in photorefractive crystals. Stability of the single-component vortex cross in the anti-continuum limit of small coupling between lattice nodes is proved. In the vector case, we consider two coupled configurations of vortex crosses, namely the charge-one vortex in one component coupled in the other component to either the charge-one vortex (forming a double-charge vortex) or the charge-negative-one vortex (forming a, so-called, hidden-charge vortex). We show that both vortex configurations are stable in the anti-continuum limit if the parameter for the inter-component coupling is small and both of them are unstable when the coupling parameter is large. In the marginal case of the discrete two-dimensional Manakov system, the double-charge vortex is stable while the hidden-charge vortex is linearly unstable. Analytical predictions are corroborated with numerical observations that show good agreement near the anti-continuum limit but gradually deviate for larger couplings between the lattice nodes.
\end{abstract}

\section{Introduction}

In the past few years, the developments in the nonlinear optics of photorefractive materials 1] and of Bose-Einstein condensates in optical lattices [2, 3, 4, have stimulated an enormous amount of theoretical, numerical and experimental activity in the area of discrete nonlinear Hamiltonian systems. A particular focus in this effort has been drawn to the prototypical lattice model of the discrete nonlinear Schrödinger (DNLS) equation [5]. The latter, either as a tight binding limit [6], or as a generic discrete nonlinear envelope wave equation [7] plays a key role in unveiling the relevant dynamics within the appropriate length and time scales.

One of the principal directions of interest in these lattice systems consists of the effort to analyze the main features of their localized solutions. In the particular case of two spatial dimensions, such structures can be regular discrete solitons [8] or discrete vortices (i.e., struc- 
tures that have topological charge over a discrete contour) 9]. The study of these types of coherent structures has made substantial leaps of progress in the past two years with the numerical and experimental observation of regular discrete solitons [10, 11], dipole solitons [12, soliton-trains [13], soliton-necklaces [14] and vector solitons [15] in photorefractive crystals and experimental discovery of robust discrete vortex states [16, 17, based on earlier theoretical predictions [18, 19, 20].

On the other hand, the recent years were marked by the experimental developments in soft condensed-matter physics of Bose-Einstein Condensates (BECs). Among the important recent observations one can single out the experimental illustration of the dark [21, 22, 23], bright [24, 25] and gap [26] solitons in quasi-one dimensional BECs. The experimental capabilities seem to be on the verge of producing similar structures in a two-dimensional context [27].

In both of the above contexts (nonlinear optics and atomic physics), multi-component systems were recently studied due to their relevance to applications. In particular, the first observations of discrete vector solitons in nonlinear waveguide arrays were reported in [28, 29], while numerous experiments with BECs were directed towards studies of mixtures of different spin states of ${ }^{87} \mathrm{Rb}$ [30, 31] or ${ }^{23} \mathrm{Na}$ 32] and even ones of different atomic species such as ${ }^{41} \mathrm{~K}-{ }^{87} \mathrm{Rb}$ 33 and ${ }^{7} \mathrm{Li}^{-133} \mathrm{Cs}$ 34]. While the above BEC experiments did not include the presence of an optical lattice, the addition of an external optical potential could be manufactured within the present experimental capabilities [4].

It is the purpose of the present work to address these recent features of the physical experiments, namely discrete systems with multiple components. In particular, we aim at addressing the fundamental issue of how localized excitations are affected by the presence of two components which are coupled (nonlinearly) to each other. While our results will be presented for the specific example of two coupled DNLS equations with cubic nonlinearities, we believe that similar features persist in a variety of other models. We should note here that rather few studies have focused on the two-dimensional vector generalization of the DNLS equation 35, 36, 37. Among others, we mention the work 38] which was motivated by the experimental system of the nonlinear waveguide arrays proposed in 29]. To the best of our knowledge, these earlier studies did not address vortices in coupled discrete systems.

For vortices in coupled systems, a number of interesting questions emerges concerning the stability of particular vortex configurations (e.g. the so-called vortex cross [16, 17]) including the case of equal charges in both components and the case of opposite charges between the two components. The former state has a double vortex charge, while the latter has a hidden vortex charge. It has been shown for the continuous NLS equation with cubic-quintic [39] and saturable [40] nonlinearities that these two states have different stability windows.

In the present setting, we examine the stability of such vortex structures in the discrete case both analytically and numerically. We use the method of Lyapunov-Schmidt reductions 
developed earlier in 9]. This method allows for direct analytical calculations of eigenvalues of the linear stability problem as functions of the system parameters (such as the coupling between adjacent lattice sites and the coupling between the two components).

Our presentation is structured as follows. In section 2, we introduce the setup and the vortex cross configurations. In section 3, we study the stability of such configurations in the one-component model. In section 4, we generalize the vortex cross configuration to the twocomponent case and compare our results with numerical computations of the parameter continuations. In section 5, we deal with a special Manakov case of the system of two DNLS equations. Finally, in section 6, we summarize our findings. Appendix A presents technical details for the case of the single-component vortex cross.

\section{Setup}

We write the coupled system of discrete nonlinear Schrödinger (DNLS) equations in the form:

$$
\begin{aligned}
i \dot{u}_{n, m}+\epsilon\left(u_{n+1, m}+u_{n-1, m}+u_{n, m+1}+u_{n, m-1}\right)+\left(\left|u_{n, m}\right|^{2}+\beta\left|v_{n, m}\right|^{2}\right) u_{n, m} & =0 \\
i \dot{v}_{n, m}+\epsilon\left(v_{n+1, m}+v_{n-1, m}+v_{n, m+1}+v_{n, m-1}\right)+\left(\beta\left|u_{n, m}\right|^{2}+\left|v_{n, m}\right|^{2}\right) v_{n, m} & =0
\end{aligned}
$$

where $\beta$ is a non-negative parameter for the coupling between the two components $(u, v)$ and $\epsilon$ is a small non-negative parameter for the coupling between adjacent lattice sites. Localized modes of the coupled system (2.1) - (2.2) take the form:

$$
u_{n, m}(t)=\phi_{n, m} e^{i t}, \quad v_{n, m}(t)=\psi_{n, m} e^{i \omega t},
$$

where $\omega$ is a parameter of time-periodic solutions and $\left(\phi_{n, m}, \psi_{n, m}\right)$ satisfy the system of nonlinear difference equations:

$$
\begin{aligned}
& \left(1-\left|\phi_{n, m}\right|^{2}-\beta\left|\psi_{n, m}\right|^{2}\right) \phi_{n, m}=\epsilon\left(\phi_{n+1, m}+\phi_{n-1, m}+\phi_{n, m+1}+\phi_{n, m-1}\right) \\
& \left(\omega-\beta\left|\phi_{n, m}\right|^{2}-\left|\psi_{n, m}\right|^{2}\right) \psi_{n, m}=\epsilon\left(\psi_{n+1, m}+\psi_{n-1, m}+\psi_{n, m+1}+\psi_{n, m-1}\right) .
\end{aligned}
$$

We are interested in a particular vortex solution, called the vortex cross. An example of this solution is obtained numerically for $\beta=\frac{2}{3}, \omega=1$ and $\epsilon=0.1$ and it is shown on Figure 1 Let us consider the diagonal square discrete contour on the grid $(n, m) \in \mathbb{Z}^{2}$ :

$$
S^{(0)}=\{(-1,0) ;(0,-1) ;(1,0) ;(0,1)\} \subset \mathbb{Z}^{2},
$$

enumerated in the same order by $j=1,2,3,4$. We shall assume that the vortex cross of Figure 11 bifurcates from the limiting solution at the anti-continuum limit $\epsilon=0$ :

$$
\phi_{n, m}^{(0)}=\left\{\begin{array}{c}
a e^{i \theta_{j}}, \quad(n, m) \in S^{(0)} \\
0, \quad(n, m) \notin S^{(0)}
\end{array} \quad \psi_{n, m}^{(0)}=\left\{\begin{array}{c}
b e^{i \nu_{j}}, \quad(n, m) \in S^{(0)} \\
0, \quad(n, m) \notin S^{(0)}
\end{array}\right.\right.
$$



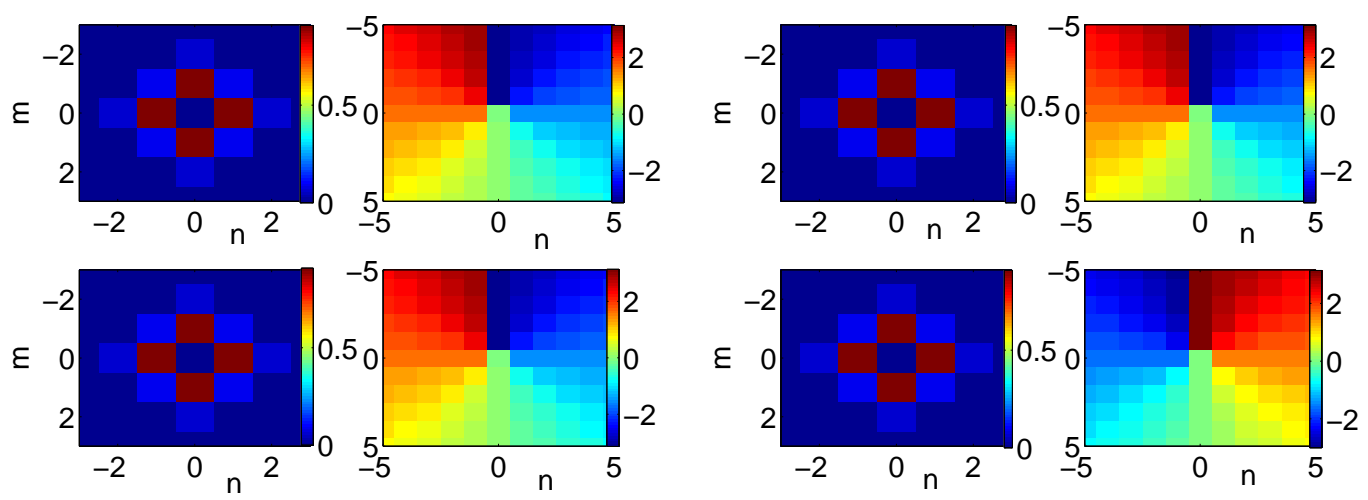

Figure 1: The contour plots show the amplitude and phase (left and right panels respectively) of the two components (top and bottom respectively) for a $(1,1)$ (left four subplots) and a $(1,-1)$ (right four subplots) vortex configuration, in the case of $\beta=2 / 3, \omega=1$, and $\epsilon=0.1$.

where the set of phase parameters $\left\{\theta_{j}, \nu_{j}\right\}_{j=1}^{4}$ is yet to be determined, while the set of amplitude parameters $(a, b)$ is determined from solutions of the system:

$$
a^{2}+\beta b^{2}=1, \quad \beta a^{2}+b^{2}=\omega
$$

When $\beta \neq 1$, there exists a unique solution of the system (2.8):

$$
a^{2}=\frac{1-\beta \omega}{1-\beta^{2}}, \quad b^{2}=\frac{\omega-\beta}{1-\beta^{2}} .
$$

The solution is meaningful only if $a^{2}>0$ and $b^{2}>0$, which define the domain of existence:

$$
\min \left(\beta, \beta^{-1}\right) \leq \omega \leq \max \left(\beta, \beta^{-1}\right) .
$$

When $\beta=1$, the domain of existence shrinks into the line $\omega=1$ and the solution of the system (2.8) forms a one-parameter family:

$$
a=\cos \delta, \quad b=\sin \delta, \quad \delta \in[0,2 \pi]
$$

The vortex cross, if it exists, is defined by the phase configurations along the discrete contour $S^{(0)}$ :

$$
\theta_{j}=\frac{\pi(j-1)}{2}, \quad \nu_{j}= \pm \frac{\pi(j-1)}{2}, \quad j=1,2,3,4 .
$$

The upper sign corresponds to the $(1,1)$ coupled state called the double-charge vortex, while the lower sign corresponds to the $(1,-1)$ coupled state called the hidden-charge vortex. Persistence and stability of the vortex configurations (2.7), (2.9), and (2.12) are addressed separately in the cases $\beta=0,0<\beta<1, \beta=1$, and $\beta>1$. 


\section{Scalar vortex cross}

We apply the method of Lyapunov-Schmidt (LS) reductions developed in [9] to the scalar nonlinear difference equation:

$$
\left(1-\left|\Phi_{n, m}\right|^{2}\right) \Phi_{n, m}=\epsilon\left(\Phi_{n+1, m}+\Phi_{n-1, m}+\Phi_{n, m+1}+\Phi_{n, m-1}\right)
$$

This scalar equation corresponds to the reduction $\psi_{n, m}=0, \forall(n, m) \in \mathbb{Z}^{2}$ of the system (2.4) - 2.5). Local existence of a single-component vortex cross in the scalar problem (3.1) is proved in Appendix A for small values of $\epsilon$ (on the basis of Proposition 2.9 in [9]). This result is formulated as follows.

Proposition 3.1 There exists a unique (up to the gauge invariance) continuation in $\epsilon$ of the limiting solution at $\epsilon=0$ :

$$
\Phi_{n, m}^{(0)}=\left\{\begin{array}{l}
e^{i \theta_{j}}, \quad(n, m) \in S^{(0)} \\
0, \quad(n, m) \notin S^{(0)}
\end{array}\right.
$$

where $S^{(0)}$ is given by (2.6) and the values of $\theta_{j}$ are given by (2.12). The family of vortex solutions $\Phi_{n, m}(\epsilon),(n, m) \in \mathbb{Z}^{2}$ is a smooth (real analytic) function of $\epsilon$.

To address spectral stability of the vortex cross in the time-evolution of the single-component DNLS equation, we consider the linearization problem with the explicit formula

$$
u_{n, m}(t)=e^{i t}\left[\Phi_{n, m}+a_{n, m} e^{\lambda t}+\bar{b}_{n, m} e^{\bar{\lambda} t}\right],
$$

and derive the linear eigenvalue problem from the DNLS equation,

$$
\begin{gathered}
\left(1-2\left|\Phi_{n, m}\right|^{2}\right) a_{n, m}-\Phi_{n, m}^{2} b_{n, m}-\epsilon\left(a_{n+1, m}+a_{n-1, m}+a_{n, m+1}+a_{n, m-1}\right)=i \lambda a_{n, m} \\
\left(1-2\left|\Phi_{n, m}\right|^{2}\right) b_{n, m}-\bar{\Phi}_{n, m}^{2} a_{n, m}-\epsilon\left(b_{n+1, m}+b_{n-1, m}+b_{n, m+1}+b_{n, m-1}\right)=-i \lambda b_{n, m},
\end{gathered}
$$

where $\lambda$ is an eigenvalue and $\left(a_{n, m}, b_{n, m}\right)$ are components of an eigenvector. Symbolically, we write the linear eigenvalue problem as

$$
\mathcal{H}(\epsilon) \varphi=i \lambda \sigma \varphi
$$

where $\mathcal{H}(\epsilon)$ is the linearized Jacobian matrix for the system (3.1), $\sigma$ is a diagonal matrix of $(1,-1)$, and $\boldsymbol{\varphi}$ is an eigenvector consisting of $\left(a_{n, m}, b_{n, m}\right)$. The linear eigenvalue problem for the limiting solution $\Phi_{n, m}=\Phi_{n, m}^{(0)}$ at $\epsilon=0$ has a set of double zero eigenvalues with the eigenvectors $\mathbf{e}_{j}$ and generalized eigenvectors $\hat{\mathbf{e}}_{j}$, such that $\mathcal{H}^{(0)} \mathbf{e}_{j}=\mathbf{0}$ and $\mathcal{H}^{(0)} \hat{\mathbf{e}}_{j}=2 i \sigma \mathbf{e}_{j}$, where $\mathcal{H}^{(0)}=\mathcal{H}(0)$. The index $j$ enumerates the set $S^{(0)}$ and the eigenvectors $\mathbf{e}_{j}$ and $\hat{\mathbf{e}}_{j}$ have non-zero components only at the corresponding nodes of the set $S^{(0)}$,

$$
\mathbf{e}_{j}=i\left(\begin{array}{c}
e^{i \theta_{j}} \\
-e^{-i \theta_{j}}
\end{array}\right), \quad \hat{\mathbf{e}}_{j}=\left(\begin{array}{c}
e^{i \theta_{j}} \\
e^{-i \theta_{j}}
\end{array}\right) .
$$


The kernel of $\mathcal{H}(\epsilon)$ for $\epsilon \neq 0$ includes at least one eigenfunction

$$
\boldsymbol{\varphi}_{n, m}=\left(\begin{array}{c}
\Phi_{n, m} \\
-\bar{\Phi}_{n, m}
\end{array}\right), \quad(n, m) \in \mathbb{Z}^{2},
$$

which follows from the gauge invariance of the DNLS equation with respect to rotation of the complex phase in $\Phi_{n, m},(n, m) \in \mathbb{Z}^{2}$. It is easy to show that a generalized kernel for zero eigenvalue is non-empty as it includes a solution of the inhomogeneous equation $\mathcal{H}(\epsilon) \tilde{\varphi}=2 i \sigma \varphi$ exists, where $\varphi$ is given by (3.7).

Using the perturbation series expansion for $\Phi_{n, m}(\epsilon)$, we define the expansion $\mathcal{H}(\epsilon)=\mathcal{H}^{(0)}+$ $\epsilon \mathcal{H}^{(1)}+\epsilon^{2} \mathcal{H}^{(2)}+\mathrm{O}\left(\epsilon^{3}\right)$. By Lemma 4.1 in [9], computations of Appendix A determine the splitting of zero eigenvalues of $\mathcal{H}(\epsilon)$ as $\epsilon \neq 0$. The splitting of zero eigenvalues of $\sigma \mathcal{H}(\epsilon)$ is formulated and proved as follows.

Proposition 3.2 Let $\Phi_{n, m}(\epsilon),(n, m) \in \mathbb{Z}^{2}$ be a family of vortex solutions defined by Proposition [3.1. The linearized problem (3.3)-3.4) has zero eigenvalue of algebraic multiplicity two and geometric multiplicity one and three small pairs of purely imaginary eigenvalues of negative Krein signatures ${ }^{1}$ with the asymptotic approximations,

$$
\lambda_{1,2}, \lambda_{3,4}= \pm 2 i \epsilon+\mathrm{O}\left(\epsilon^{2}\right), \quad \lambda_{5,6}= \pm 4 i \epsilon^{2}+\mathrm{O}\left(\epsilon^{3}\right) .
$$

The rest of the spectrum is bounded away the origin as $\epsilon \rightarrow 0$ and it is located on the imaginary axis of $\lambda$.

Proof. We supplement the general proof of Lemma 4.2 in [9] with the explicit perturbation series expansions for small eigenvalues of the linear eigenvalue problem (3.5):

$$
\boldsymbol{\varphi}=\boldsymbol{\varphi}^{(0)}+\epsilon \boldsymbol{\varphi}^{(1)}+\epsilon^{2} \boldsymbol{\varphi}^{(2)}+\mathrm{O}\left(\epsilon^{3}\right), \quad \lambda=\epsilon \lambda_{1}+\epsilon^{2} \lambda_{2}+\mathrm{O}\left(\epsilon^{3}\right),
$$

where

$$
\boldsymbol{\varphi}^{(0)}=\sum_{j=1}^{4} c_{j} \mathbf{e}_{j}, \quad \boldsymbol{\varphi}^{(1)}=\frac{\lambda_{1}}{2} \sum_{j=1}^{4} c_{j} \hat{\mathbf{e}}_{j}+\boldsymbol{\varphi}_{\text {inhom }}^{(1)},
$$

and the solution $\varphi_{\text {inhom }}^{(1)}=-\mathcal{H}^{(0)-1} \mathcal{H}^{(1)} \boldsymbol{\varphi}^{(0)}=-\mathcal{H}^{(1)} \boldsymbol{\varphi}^{(0)}$ is uniquely defined on the set $S^{(1)}$, where $S^{(1)}$ is the set of adjacent nodes to the set of $S^{(0)}$. At the second-order perturbation theory, the problem is written in the form,

$$
\mathcal{H}^{(0)} \boldsymbol{\varphi}^{(2)}+\mathcal{H}^{(1)} \boldsymbol{\varphi}^{(1)}+\mathcal{H}^{(2)} \boldsymbol{\varphi}^{(0)}=i \lambda_{1} \sigma \varphi^{(1)}+i \lambda_{2} \sigma \varphi^{(0)} .
$$

Projecting the problem to the kernel of $\mathcal{H}^{(0)}$, we find the reduced eigenvalue problem:

$$
\mathcal{M}_{2} \mathbf{c}=\frac{1}{2} \lambda_{1}^{2} \mathbf{c}
$$

\footnotetext{
${ }^{1} \mathrm{~A}$ simple eigenvalue of the linear eigenvalue problem (3.5) is said to have the negative Krein signature if the quadratic form for the associated eigenvector $(\mathcal{H}(\epsilon) \varphi, \varphi)$ is negative.
} 

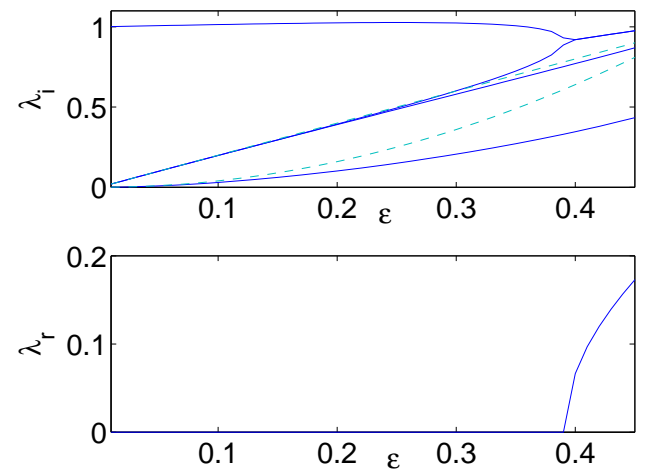

Figure 2: Eigenvalues of the scalar vortex cross versus $\epsilon$. The top panel shows the imaginary part of the relevant eigenvalues, while the bottom panel shows the real part. The solid lines display the numerical results, while the dashed ones correspond to the asymptotic approximations.

where $\mathbf{c}=\left(c_{1}, c_{2}, c_{3}, c_{4}\right)^{T}$ and $\mathcal{M}_{2}$ is computed in Appendix A. Therefore, two negative eigenvalues $\gamma_{1,2}$ of the Jacobian matrix $\epsilon^{2} \mathcal{M}_{2}$ generate two pairs of imaginary eigenvalues of negative Krein signatures in the linear eigenvalue problem by virtue of the relation $\lambda= \pm \sqrt{2 \gamma}$. The same computation is then extended up to the fourth order, where it is found that the negative eigenvalue $\gamma_{3}$ of the extended matrix $\epsilon^{2} \mathcal{M}_{2}+\epsilon^{4} \mathcal{M}_{4}$ determines the third pair of purely imaginary eigenvalues by virtue of the same relation $\lambda= \pm \sqrt{2 \gamma}$.

We note that the count of eigenvalues of negative Krein signatures corresponds to the closure theorem for negative index of $\mathcal{H}(\epsilon)$ (see [8] for details). There are four negative eigenvalues of $\mathcal{H}^{(0)}$ for the limiting solution (3.2) and three more small negative eigenvalues occur for $\epsilon \neq 0$. The total number of negative eigenvalues is reduced by the gauge symmetry constraint, such that six negative eigenvalues in a constrained subspace match three pairs of imaginary eigenvalues with negative Krein signature.

The asymptotic approximations of eigenvalues $\lambda$ are plotted on Figure 2 by dashed lines. The numerical computations of the same eigenvalues (up to the prescribed numerical accuracy) versus $\epsilon$ are shown by solid lines. All three pairs of purely imaginary eigenvalues bifurcate into complex domain when they collide to other eigenvalues of stability problem (e.g. with eigenvalues of positive Krein signatures or with the spectral band). The first collision is numerically detected to occur at $\epsilon \approx 0.395$. 


\section{Vector vortex crosses for $0<\beta<1$ and $\beta>1$}

In order to consider the coupled vortex configurations in the non-degenerate case $\beta \neq 1$, we extend computations of Appendix A to the solution of the coupled nonlinear difference equations (2.4)-(2.5). We report here computations for two related problems: (i) bifurcations of small eigenvalues of the linearized Jacobian matrix near the zero eigenvalue and (ii) bifurcations of small eigenvalues of the linearized stability problem near the origin. Because of the computational complexity of the analytical approximations, we shall complement the analytical results of the second-order Lyapunov-Schmidt (LS) reductions with the symbolic computational results of the fourth-order LS reductions.

Similarly to the scalar case, the linearized stability problem for the two-component system takes the matrix-vector form:

$$
\mathcal{H}(\epsilon) \varphi=i \lambda \sigma \varphi
$$

where $\mathcal{H}(\epsilon)$ is the linearized Jacobian matrix for the system (2.4)-(2.5), $\sigma$ is a diagonal matrix of $(1,-1,1,-1)$, and $\varphi$ is an eigenvector consisting of four elements of the perturbation vector at each node $(n, m) \in \mathbb{Z}^{2}$. The diagonal block of the matrix $\mathcal{H}(\epsilon)$ at each node $(n, m) \in \mathbb{Z}^{2}$ takes the form:

$$
\left(\begin{array}{cccc}
1-2\left|\phi_{n, m}\right|^{2}-\beta\left|\psi_{n, m}\right|^{2} & -\phi_{n, m}^{2} & -\beta \phi_{n, m} \bar{\psi}_{n, m} & -\beta \phi_{n, m} \psi_{n, m} \\
-\bar{\phi}_{n, m}^{2} & 1-2\left|\phi_{n, m}\right|^{2}-\beta\left|\psi_{n, m}\right|^{2} & -\beta \bar{\phi}_{n, m} \bar{\psi}_{n, m} & -\beta \bar{\phi}_{n, m} \psi_{n, m} \\
-\beta \bar{\phi}_{n, m} \psi_{n, m} & -\beta \phi_{n, m} \psi_{n, m} & \omega-\beta\left|\phi_{n, m}\right|^{2}-2\left|\psi_{n, m}\right|^{2} & -\psi_{n, m}^{2} \\
-\beta \bar{\phi}_{n, m} \bar{\psi}_{n, m} & -\beta \phi_{n, m} \bar{\psi}_{n, m} & -\bar{\psi}_{n, m}^{2} & \omega-\beta\left|\phi_{n, m}\right|^{2}-2\left|\psi_{n, m}\right|^{2}
\end{array}\right)
$$

The non-diagonal blocks of $\mathcal{H}(\epsilon)$ comes from the difference operators in the right-hand-side of the system (2.4)-(2.5).

\subsection{Bifurcations of zero eigenvalues of the linearized Jacobian matrix}

We extend the perturbation series expansions (A.1) to the two-component case,

$$
\phi_{n, m}(\epsilon)=\sum_{k=0}^{\infty} \epsilon^{k} \phi_{n, m}^{(k)}, \quad \psi_{n, m}(\epsilon)=\sum_{k=0}^{\infty} \epsilon^{k} \psi_{n, m}^{(k)}
$$

where the zero-order solution in the anti-continuum limit is given by (2.7) and parameters $(a, b)$ are given in (2.9). The first-order corrections are found from the uncoupled system of equations, similarly to the scalar case:

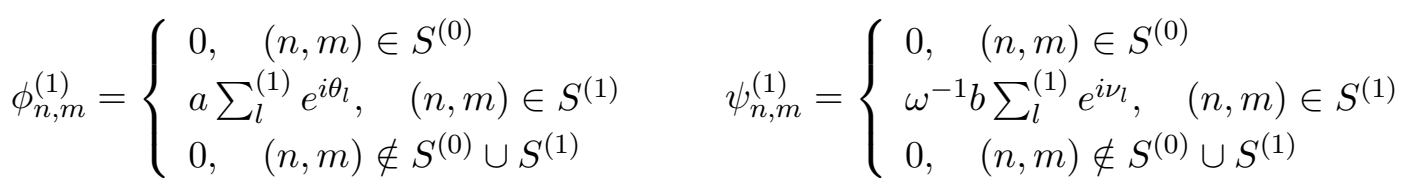


where $\sum_{l}^{(1)}$ is defined in (A.3). The second-order corrections are found in the form:

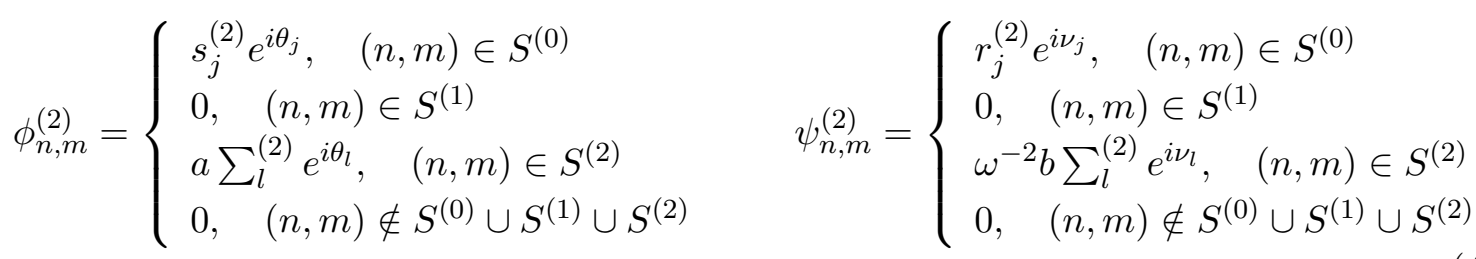

where $\sum_{l}^{(2)}$ is defined in (A.5). The real parameters $\left(s_{j}^{(2)}, r_{j}^{(2)}\right)$ satisfy an inhomogeneous system

$$
\begin{aligned}
-2\left(a s_{j}^{(2)}+\beta b r_{j}^{(2)}\right) & =4+2 \cos \left(\theta_{j+1}-\theta_{j}\right)+2 \cos \left(\theta_{j-1}-\theta_{j}\right)+\cos \left(\theta_{j+2}-\theta_{j}\right), \\
-2\left(\beta a s_{j}^{(2)}+b r_{j}^{(2)}\right) & =4+2 \cos \left(\nu_{j+1}-\nu_{j}\right)+2 \cos \left(\nu_{j-1}-\nu_{j}\right)+\cos \left(\nu_{j+2}-\nu_{j}\right) .
\end{aligned}
$$

When $\beta \neq 1$, the inhomogeneous system (4.5)-(4.6) has a unique solution. Second-order corrections to the bifurcation equations are uncoupled and have the form:

$$
\begin{aligned}
& g_{j}^{(2)}=2 \sin \left(\theta_{j}-\theta_{j+1}\right)+2 \sin \left(\theta_{j}-\theta_{j-1}\right)+\sin \left(\theta_{j}-\theta_{j+2}\right), \\
& h_{j}^{(2)}=2 \sin \left(\nu_{j}-\nu_{j+1}\right)+2 \sin \left(\nu_{j}-\nu_{j-1}\right)+\sin \left(\nu_{j}-\nu_{j+2}\right),
\end{aligned}
$$

where a suitable normalization of $g_{j}^{(2)}$ and $h_{j}^{(2)}$ is made. As a result, the Jacobian matrix computed from derivatives of $\left(g_{j}^{(2)}, h_{j}^{(2)}\right)^{T}$ in $\left(\theta_{i}, \nu_{i}\right)$ is block-diagonal as $\operatorname{diag}\left(\mathcal{M}_{2}, \mathcal{M}_{2}\right)$, where $\mathcal{M}_{2}$ is defined in Appendix A. By Lemma 4.1 in [9], non-zero eigenvalues of $\operatorname{diag}\left(\mathcal{M}_{2}, \mathcal{M}_{2}\right)$ determine small eigenvalues of the linearized Jacobian matrix $\mathcal{H}(\epsilon)$,

$$
\gamma_{1,2,3,4}=-2 \epsilon^{2}+\mathrm{O}\left(\epsilon^{4}\right)
$$

Two zero eigenvalues of $\operatorname{diag}\left(\mathcal{M}_{2}, \mathcal{M}_{2}\right)$ split into two non-zero eigenvalues in the fourth-order LS reductions, while two other zero eigenvalues of $\operatorname{diag}\left(\mathcal{M}_{2}, \mathcal{M}_{2}\right)$ persist beyond all orders due to the gauge invariance of each component in the the coupled DNLS equations (2.1)-(2.2). Indeed, the kernel of $\mathcal{H}(\epsilon)$ for $\epsilon \neq 0$ includes at least two eigenfunctions:

$$
\boldsymbol{\varphi}_{n, m}=\left\{\left(\begin{array}{c}
\phi_{n, m} \\
-\bar{\phi}_{n, m} \\
0 \\
0
\end{array}\right),\left(\begin{array}{c}
0 \\
0 \\
\psi_{n, m} \\
-\bar{\psi}_{n, m}
\end{array}\right)\right\}, \quad(n, m) \in \mathbb{Z}^{2}
$$

In order to compute the small non-zero eigenvalues of the linearized Jacobian matrix $\mathcal{H}(\epsilon)$, we use the symbolic computation package based on Wolfram's Mathematica ${ }^{2}$. The projection

\footnotetext{
${ }^{2}$ The software programs are available online at http://dmpeli.math.mcmaster.ca/Software/LSreductions.html For more information on the symbolic mathematics package in which these programs were implemented, see http://www.wolfram.com
} 
to the eigenspace of $\operatorname{diag}\left(\mathcal{M}_{2}, \mathcal{M}_{2}\right)$ spanned by eigenvectors $\left(\mathbf{p}_{2}, \mathbf{0}_{4}\right)^{T}$ and $\left(\mathbf{0}_{4}, \mathbf{p}_{2}\right)^{T}$, where $\mathbf{p}_{2}=(-1,1,-1,1)$ and $\mathbf{0}_{4}=(0,0,0,0)$, leads to the reduced eigenvalue problem (for $\left.\omega=1\right)$,

$$
\begin{aligned}
\frac{-8}{1+\beta}\left(\alpha_{1} \pm \beta \alpha_{2}\right) & =\tilde{\gamma} \alpha_{1} \\
\frac{-8}{1+\beta}\left( \pm \beta \alpha_{1}+\alpha_{2}\right) & =\tilde{\gamma} \alpha_{2}
\end{aligned}
$$

where $\left(\alpha_{1}, \alpha_{2}\right)$ are coordinates of the projections, $\tilde{\gamma}=\lim _{\epsilon \rightarrow 0} \epsilon^{-4} \gamma$, and the upper/lower signs refer to the two coupled vortices $(1, \pm 1)$. It is clear that the eigenvalues of the reduced eigenvalue problem are the same for either sign and they define two small eigenvalues of the linearized Jacobian matrix $\mathcal{H}(\epsilon)$ (for $\omega=1$ ):

$$
\gamma_{5}=-8 \epsilon^{4}+\mathrm{O}\left(\epsilon^{6}\right), \quad \gamma_{6}=-\frac{8(1-\beta)}{(1+\beta)} \epsilon^{4}+\mathrm{O}\left(\epsilon^{6}\right)
$$

\subsection{Bifurcations of zero eigenvalues of the linearized stability problem}

We consider the eigenvalue problem (4.1) in the limit of small $\epsilon$. Let $\mathcal{H}(\epsilon)=\mathcal{H}^{(0)}+\epsilon \mathcal{H}^{(1)}+$ $\epsilon^{2} \mathcal{H}^{(2)}+\mathrm{O}\left(\epsilon^{3}\right)$. The set of eigenvectors of $\mathcal{H}^{(0)} \mathbf{e}_{j}=\mathbf{0}$ and $\mathcal{H}^{(0)} \mathbf{f}_{j}=\mathbf{0}$ takes the form:

$$
\mathbf{e}_{j}=i\left(\begin{array}{c}
e^{i \theta_{j}} \\
-e^{-i \theta_{j}} \\
0 \\
0
\end{array}\right), \quad \mathbf{f}_{j}=i\left(\begin{array}{c}
0 \\
0 \\
e^{i \nu_{j}} \\
-e^{-i \nu_{j}}
\end{array}\right)
$$

The corresponding set of generalized eigenvectors of $\mathcal{H}^{(0)} \hat{\mathbf{e}}_{j}=2 i \sigma \mathbf{e}_{j}$ and $\mathcal{H}^{(0)} \hat{\mathbf{f}}_{j}=2 i \sigma \mathbf{f}_{j}$ takes the form:

$$
\hat{\mathbf{e}}_{j}=\left(\begin{array}{c}
A_{+} e^{i \theta_{j}} \\
A_{+} e^{-i \theta_{j}} \\
B_{+} e^{i \nu_{j}} \\
B_{+} e^{-i \nu_{j}}
\end{array}\right), \quad \mathbf{f}_{j}=\left(\begin{array}{c}
A_{-} e^{i \theta_{j}} \\
A_{-} e^{-i \theta_{j}} \\
B_{-} e^{i \nu_{j}} \\
B_{-} e^{-i \nu_{j}}
\end{array}\right)
$$

where

$$
A_{+}=\frac{1}{a^{2}\left(1-\beta^{2}\right)}, \quad B_{+}=A_{-}=\frac{-\beta}{a b\left(1-\beta^{2}\right)}, \quad B_{-}=\frac{1}{b^{2}\left(1-\beta^{2}\right)} .
$$

Bifurcations of zero eigenvalues of the linear eigenvalue problem (4.1) can be computed with the extended perturbation series expansions (4.2) for $\phi_{n, m}(\epsilon)$ and $\psi_{n, m}(\epsilon)$ and extended perturbation series (3.8) for $\varphi$ and $\lambda$, where

$$
\boldsymbol{\varphi}^{(0)}=\sum_{j=1}^{4} c_{j} \mathbf{e}_{j}+\sum_{j=1}^{4} d_{j} \mathbf{f}_{j}, \quad \boldsymbol{\varphi}^{(1)}=\frac{\lambda_{1}}{2} \sum_{j=1}^{4} c_{j} \hat{\mathbf{e}}_{j}+\frac{\lambda_{1}}{2} \sum_{j=1}^{4} d_{j} \hat{\mathbf{f}}_{j}+\boldsymbol{\varphi}_{\text {inhom }}^{(1)}
$$


and $\varphi_{\text {inhom }}^{(1)}=-\mathcal{H}^{(0)-1} \mathcal{H}^{(1)} \varphi^{(0)}=-\mathcal{H}^{(1)} \varphi^{(0)}$ is uniquely defined on the set $S^{(1)}$. At the second-order perturbation theory, we have the same problem (3.9), from which we derive the reduced eigenvalue problem:

$$
\begin{aligned}
\mathcal{M}_{2} \mathbf{c} & =\frac{1}{2} \lambda_{1}^{2}\left(A_{+} \mathbf{c}+A_{-} \mathbf{d}\right) \\
\mathcal{M}_{2} \mathbf{d} & =\frac{1}{2} \lambda_{1}^{2}\left(B_{+} \mathbf{c}+B_{-} \mathbf{d}\right),
\end{aligned}
$$

where $\mathbf{c}=\left(c_{1}, c_{2}, c_{3}, c_{4}\right)^{T}, \mathbf{d}=\left(d_{1}, d_{2}, d_{3}, d_{4}\right)^{T}$, and $\mathcal{M}_{2}$ is the same as in the scalar case. Let $\gamma_{1}=\frac{1}{2} \lambda_{1}^{2}$. The reduced eigenvalue problem (4.10) (4.11) has four zero roots for $\gamma_{1}$ and two double-degenerate non-zero roots for $\gamma_{1}$, given from the quadratic equation:

$$
\left(\gamma_{1}+2 a^{2}\right)\left(\gamma_{1}+2 b^{2}\right)=4 a^{2} b^{2} \beta^{2}
$$

If $\omega=1$, such that $a^{2}=b^{2}=\frac{1}{1+\beta}$, then the two non-zero roots for $\gamma_{1}$ are found explicitly,

$$
\gamma_{ \pm}=-\frac{2(1 \mp|\beta|)}{1+\beta}
$$

By using the relation $\lambda_{1}= \pm \sqrt{2 \gamma_{1}}$, we have just proved that the linear eigenvalue problem (4.1) in the case $\omega=1$ and $0<\beta<1$ has four small pairs of purely imaginary eigenvalues with asymptotic approximations:

$$
\lambda_{1,2}, \lambda_{3,4}= \pm 2 i \epsilon+\mathrm{O}\left(\epsilon^{2}\right), \quad \lambda_{5,6}, \lambda_{7,8}= \pm 2 i \epsilon \sqrt{\frac{1-\beta}{1+\beta}}+\mathrm{O}\left(\epsilon^{2}\right) .
$$

Two pairs of eigenvalues $\lambda_{5,6}$ and $\lambda_{7,8}$ become pairs of real eigenvalues in the case $\beta>1$. Two pairs of zero eigenvalues of the reduced eigenvalue problem (4.10)-(4.11) split at the fourth-order LS reductions as pairs of non-zero eigenvalues $\lambda_{9,10}$ and $\lambda_{11,12}$. Two other pairs of zero eigenvalues persist beyond all orders for $\epsilon \neq 0$ since the geometric kernel includes two explicit solutions (4.7)) and there exists a two-parameter solution of the inhomogeneous equation $\mathcal{H}(\epsilon) \tilde{\varphi}=2 i \sigma \varphi$, where $\varphi$ is given by (4.7). In order to find the small non-zero pairs of eigenvalues, we apply again the symbolic computation package based on Wolfram's Mathematica. The projection to the eigenspace of $\operatorname{diag}\left(\mathcal{M}_{2}, \mathcal{M}_{2}\right)$ spanned by eigenvectors $\left(\mathbf{p}_{2}, \mathbf{0}_{4}\right)^{T}$ and $\left(\mathbf{0}_{4}, \mathbf{p}_{2}\right)^{T}$ for $\lambda_{1}=0$ leads to the reduced eigenvalue problem (for $\omega=1$ ),

$$
\begin{aligned}
\frac{-8}{1+\beta}\left(\alpha_{1} \pm \beta \alpha_{2}\right) & =\frac{1}{2(1-\beta)} \lambda_{2}^{2}\left(\alpha_{1}-\beta \alpha_{2}\right) \\
\frac{-8}{1+\beta}\left( \pm \beta \alpha_{1}+\alpha_{2}\right) & =\frac{1}{2(1-\beta)} \lambda_{2}^{2}\left(-\beta \alpha_{1}+\alpha_{2}\right),
\end{aligned}
$$

where $\left(\alpha_{1}, \alpha_{2}\right)$ are coordinates of the projections and the upper/lower signs refer to the two coupled vortices $(1, \pm 1)$. The eigenvalues of the reduced eigenvalue problem differs between the double-charge vortex $(1,1)$ and the hidden-charge vortex $(1,-1)$. For the double-charge vortex, 
the two pairs of small eigenvalues of the linearized stability problem are purely imaginary for any $\beta$ :

$$
(1,1): \quad \lambda_{9,10}= \pm 4 i \epsilon^{2}+\mathrm{O}\left(\epsilon^{3}\right), \quad \lambda_{11,12}= \pm 4 i\left|\frac{1-\beta}{1+\beta}\right| \epsilon^{2}+\mathrm{O}\left(\epsilon^{3}\right) .
$$

For the hidden-charge vortex, the two pairs of small eigenvalues of the linearized stability problem are purely imaginary for $0<\beta<1$ and real for $\beta>1$ :

$$
(1,-1): \quad \lambda_{9,10}, \lambda_{11,12}= \pm 4 i \sqrt{\frac{1-\beta}{1+\beta}} \epsilon^{2}+\mathrm{O}\left(\epsilon^{3}\right) .
$$

We can specify precisely how many purely imaginary eigenvalues of the linearized stability problem (4.1) have negative Krein signature. When $0<\beta<1$, there are eight negative eigenvalues of $\mathcal{H}^{(0)}$ for the limiting solution (2.7) and six more small negative eigenvalues occur for $\epsilon \neq 0$. The total number of negative eigenvalues is reduced by two gauge symmetry constraints, such that twelve negative eigenvalues in a constrained subspace match six pairs of imaginary eigenvalues with negative Krein signature. When $\beta>1$, there are four negative eigenvalues of $\mathcal{H}^{(0)}$ for the limiting solution (2.7) and five more small negative eigenvalues occur for $\epsilon \neq 0$. The total number of negative eigenvalues is reduced by one ${ }^{3}$, such that eight negative eigenvalues in a constrained subspace match two real eigenvalues and three pairs of imaginary eigenvalues with negative Krein signature for the double-charge vortex and four real eigenvalues and two pairs of imaginary eigenvalues with negative Krein signature for the hidden-charge vortex. Therefore, the last pair of purely imaginary eigenvalues $\lambda_{11,12}$ for the double-charge vortex has positive Krein signature for $\beta>1$.

We obtain numerically small eigenvalues $\lambda$ for small values of $\epsilon$ and $\omega=1$. The results are shown on Figure 3 for $\beta=\frac{2}{3}$ and on Figure 4 for $\beta=2$. The left plot corresponds to the vortex pair $(1,1)$, while the right plot corresponds to the vortex pair $(1,-1)$. We note that the degeneracy of the pairs $\lambda_{1,2}=\lambda_{3,4}$ and $\lambda_{5,6}=\lambda_{7,8}$ is preserved for the case $(1,-1)$, such that each bolded curve is double. The degeneracy of these eigenvalues is broken for the case $(1,1)$ and it is also broken for the pair $\lambda_{9,10} \neq \lambda_{11,12}$ for the case $(1,-1)$.

In the case of $\beta=\frac{2}{3}$, shown in Fig. 3, all six pairs of neutrally stable eigenvalues bifurcate to the complex plane for larger values of $\epsilon$ due to the Hamiltonian-Hopf (HH) bifurcation. The first $\mathrm{HH}$ bifurcation happens earlier for the case $(1,1)$ at $\epsilon \approx 0.395$, due to the broken degeneracy between the two pairs of eigenvalues $\lambda_{1,2}$ and $\lambda_{3,4}$. For the case $(1,-1)$, the first HH bifurcation occurs at $\epsilon \approx 0.495$, i.e. the the hidden-charge vortex has a larger stability window for $0<\beta<1$ (a similar observation is reported for continuous systems in [39, 40]).

In the case of $\beta=2$, shown in Fig. 4 both cases $(1,1)$ and $(1,-1)$ are always unstable due to the pairs of eigenvalues $\lambda_{5,6}$ and $\lambda_{7,8}$. There are also additional observations. In the case

\footnotetext{
${ }^{3}$ When $\beta$ is increased from $\beta<1$ to $\beta>1$, the Hessian matrix related to two gauge symmetry constraints loses one positive eigenvalue that passes through zero at $\beta=1$ to the negative eigenvalue for $\beta>1$ [41.
} 

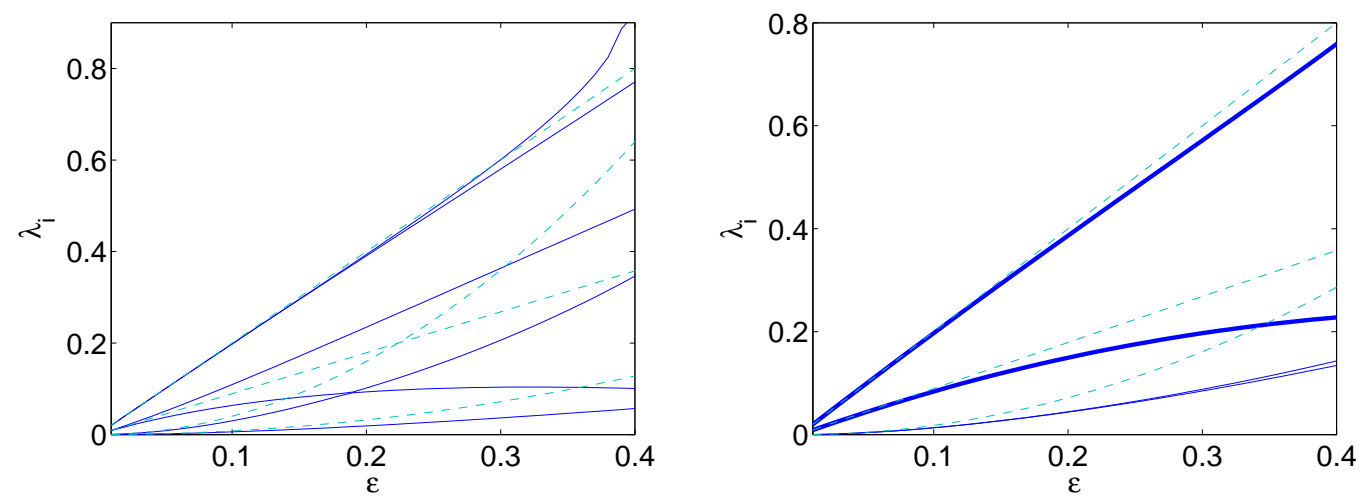

Figure 3: Eigenvalues of the vector vortex cross with $\omega=1$ and $\beta=\frac{2}{3}$ versus $\epsilon$. Left: $(1,1)$. Right: $(1,-1)$. The solid lines show the numerical results, while the dashed lines show the asymptotic approximations. Bolded curves correspond to double eigenvalues (that remain indistinguishable within the parametric window examined herein). A good agreement is observed for $\epsilon<0.1$.

$(1,1)$, the pairs of double real eigenvalues in the second-order LS reductions $\lambda_{5,6}$ and $\lambda_{7,8}$ split as a quartet of complex eigenvalues, similarly to our computations in 9. Real and imaginary parts of the quartet of complex eigenvalues are shown on Fig. 4 (left panel) by bolded curves. Only three $\mathrm{HH}$ bifurcations out of four pairs of purely imaginary eigenvalues occur for larger values of $\epsilon$. In the case $(1,-1)$, two more pairs of real eigenvalues occur such that the hiddencharge vortex is more unstable compared to the double-charge vortex for $\beta>1$. Only two HH bifurcations occur for large values of $\epsilon$.

\section{Vector vortex cross for $\beta=1$}

In the case $\beta=1$, the existence domain of the coupled vortex configurations shrinks to the line $\omega=1$. The zero-order solution in the anti-continuum limit is given by (2.7), where parameters $(a, b)$ are given by (2.11). The second-order solution of the linear inhomogeneous system (4.5)(4.6) with a singular matrix exists provided that the values of $\theta_{j}$ and $\nu_{j}$ are defined by (2.12). The arbitrary parameter in the second-order solution $s_{j}^{(2)}$ and $r_{j}^{(2)}$ renormalizes the arbitrary parameter $\delta$ in the representation (2.11).

When $\beta=\omega=1$, the existence problem (2.4) 2.5 is symmetric with respect to components $\left(\phi_{n, m}, \psi_{n, m}\right)$ such that the system (2.4) $-(2.5)$ can be reduced to the scalar difference equation (3.1) with the two independent transformations:

$$
(1,1) \quad: \quad \phi_{n, m}=\cos \delta \Phi_{n, m}, \quad \psi_{n, m}=\sin \delta \Phi_{n, m}
$$



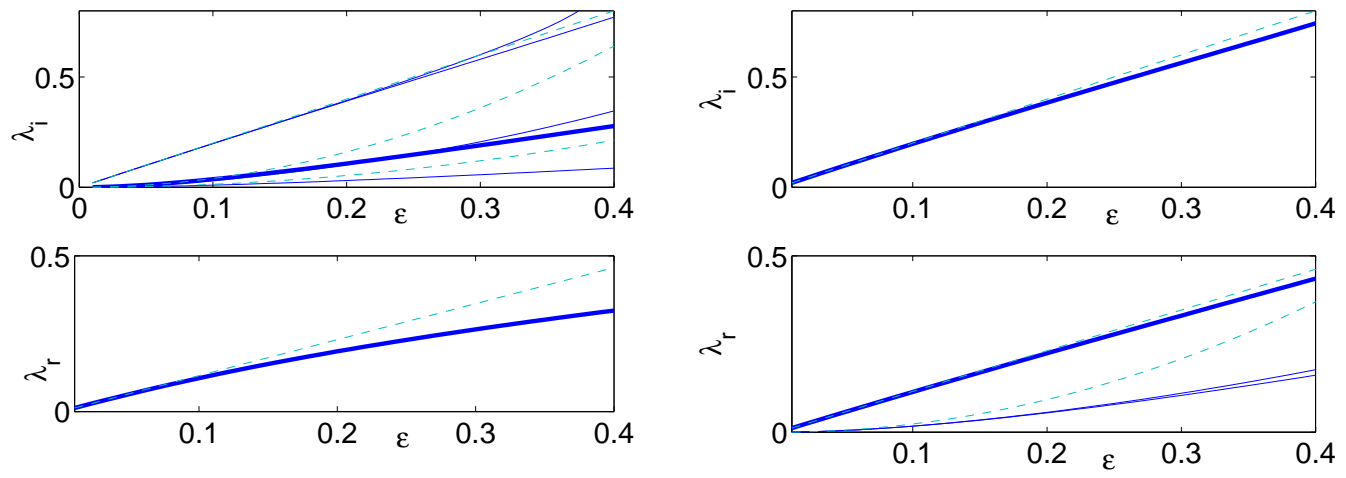

Figure 4: Eigenvalues of the vector vortex cross with $\omega=1$ and $\beta=2$ versus $\epsilon$. Left: $(1,1)$. Right: $(1,-1)$. The solid lines show the numerical results, while the dashed lines show the asymptotic approximations. Bolded curves on the left panel correspond to the real and imaginary parts of complex eigenvalues, while bolded curves on the right panel correspond to double eigenvalues.

$$
(1,-1) \quad: \quad \phi_{n, m}=\cos \delta \Phi_{n, m}, \quad \psi_{n, m}=\sin \delta \bar{\Phi}_{n, m}
$$

The existence result for the scalar vortex cross is formulated in Proposition [3.1. We will need the following non-degeneracy condition for the scalar vortex cross:

$$
\left(\sum_{(n, m) \in \mathbb{Z}^{2}}\left|\Phi_{n, m}\right|^{2}\right)^{2} \neq\left(\sum_{(n, m) \in \mathbb{Z}^{2}} \Phi_{n, m}^{2}\right)\left(\sum_{(n, m) \in \mathbb{Z}^{2}} \bar{\Phi}_{n, m}^{2}\right) .
$$

It is clear from the limiting solution (2.7) that the constraint (5.1) is satisfied for small $\epsilon$. The stability problem (4.1) is different between the cases $(1,1)$ and $(1,-1)$.

\subsection{Eigenvalues of the $(1,1)$ vortex cross}

In this case, the stability problem (4.1) is block-diagonalized under the following transformation of the four components of the vector $\varphi$ on the lattice node $(n, m) \in \mathbb{Z}^{2}$ :

$$
(1,1): \quad\left(\begin{array}{c}
a_{n, m} \\
b_{n, m} \\
c_{n, m}^{+} \\
c_{n, m}^{-}
\end{array}\right)=\left(\begin{array}{cccc}
\cos \delta & 0 & \sin \delta & 0 \\
0 & \cos \delta & 0 & \sin \delta \\
-\sin \delta & 0 & \cos \delta & 0 \\
0 & -\sin \delta & 0 & \cos \delta
\end{array}\right)\left(\begin{array}{l}
\varphi_{1} \\
\varphi_{2} \\
\varphi_{3} \\
\varphi_{4}
\end{array}\right)_{n, m} .
$$

The components $\left(a_{n, m}, b_{n, m}\right)$ satisfy the linear eigenvalue problem for scalar vortices (3.3)(3.4). The components $\left(c_{n, m}^{+}, c_{n, m}^{-}\right)$satisfy two uncoupled self-adjoint eigenvalue problems:

$$
\left(1-\left|\Phi_{n, m}\right|^{2}\right) c_{n, m}^{ \pm}-\epsilon\left(c_{n+1, m}^{ \pm}+c_{n-1, m}^{ \pm}+c_{n, m+1}^{ \pm}+c_{n, m-1}^{ \pm}\right)= \pm i \lambda c_{n, m}^{ \pm}
$$


Using the result of Proposition 3.2 and equivalent computations for the uncoupled self-adjoint problems (5.2), we prove the following result.

Proposition 5.1 Let $\Phi_{n, m}(\epsilon),(n, m) \in \mathbb{Z}^{2}$ be a family of vortex solutions defined by Proposition [3.1. The linearized problem [4.1] in the case $\beta=1$ for the $(1,1)$ vortex cross has zero eigenvalue of algebraic multiplicity six and geometric multiplicity five and five small pairs of purely imaginary eigenvalues given asymptotically by

$\lambda_{1,2}, \lambda_{3,4}= \pm 2 i \epsilon+\mathrm{O}\left(\epsilon^{2}\right), \quad \lambda_{5,6}= \pm 2 i \epsilon^{2}+\mathrm{O}\left(\epsilon^{3}\right), \quad \lambda_{7,8}= \pm 6 i \epsilon^{2}+\mathrm{O}\left(\epsilon^{3}\right), \quad \lambda_{9,10}= \pm 4 i \epsilon^{2}+\mathrm{O}\left(\epsilon^{3}\right)$.

The rest of the spectrum is bounded away the origin as $\epsilon \rightarrow 0$ and it is located on the imaginary axis of $\lambda$.

Proof. It remains to study bifurcations of zero eigenvalues in the self-adjoint problem (5.2) as $\epsilon \neq 0$. Let us define the perturbation series for the problem (5.2):

$$
\mathbf{c}^{ \pm}=\mathbf{c}^{(0)}+\epsilon \mathbf{c}^{(1)}+\epsilon^{2} \mathbf{c}^{(2)}+\mathrm{O}\left(\epsilon^{3}\right), \quad \lambda= \pm i \epsilon^{2} \lambda_{2}+\mathrm{O}\left(\epsilon^{3}\right) .
$$

The zero-order solution is spanned by unit vectors $\mathbf{e}_{j}$ at the $j$-th component that correspond to the node $(n, m) \in S^{(0)}$ :

$$
\mathbf{c}^{(0)}=\sum_{j=1}^{4} \alpha_{j} \mathbf{e}_{j} .
$$

The first-order correction $\mathbf{c}^{(1)}$ takes the form:

$$
c_{n, m}^{(1)}=\left\{\begin{array}{l}
0, \quad(n, m) \in S^{(0)} \\
\sum_{l}^{(1)} \alpha_{l}, \quad(n, m) \in S^{(1)} \\
0, \quad(n, m) \notin S^{(0)} \cup S^{(1)}
\end{array}\right.
$$

where the $\operatorname{sum} \sum_{l}^{(1)}$ is defined in (A.3). At the second-order in $\epsilon$, we find a set of non-trivial equations at the nodes $(n, m) \in S^{(0)}$ :

$$
\alpha_{j}+\alpha_{j+2}+2\left(\alpha_{j+1}+\alpha_{j-1}\right)=\lambda_{2} \alpha_{j}, \quad j=1,2,3,4 .
$$

The reduced eigenvalue problem has a double zero eigenvalue and two non-zero eigenvalues -2 and 6. Two zero eigenvalues of the problem (5.2) persist at all orders of $\epsilon$, because of the exact solutions: $c_{n, m}^{ \pm}=\Phi_{n, m}$ and $c_{n, m}^{ \pm}=\bar{\Phi}_{n, m}$.

We note that the pairs of eigenvalues $\lambda_{1,2}, \lambda_{3,4}$, and $\lambda_{9,10}$ continue the eigenvalues of the vortex cross $(1,1)$ from $\beta \neq 1$ to $\beta=1$. The pairs of eigenvalues $\lambda_{5,6}$ and $\lambda_{7,8}$ match with the zero $\mathrm{O}(\epsilon)$ corrections to the corresponding eigenvalues of the vortex cross $(1,1)$ for $\beta \neq 1$. Finally, the pair of non-zero eigenvalues $\lambda_{11,12}$ for $\beta \neq 1$ is forced to remain at the origin for $\beta=1$ due to the polarization-rotation symmetry. 
We can now specify how many purely imaginary eigenvalues $\lambda$ have negative Krein signature. When $\beta=1$, there are four negative and twelve zero eigenvalues of $\mathcal{H}^{(0)}$ for the limiting solution (2.7). Out of the twelve zero eigenvalues, three small negative eigenvalues bifurcate in the subspace for components $\left(a_{n, m}, b_{n, m}\right)$, two small positive and two small negative eigenvalues bifurcate in the subspace for components $\left(c_{n, m}^{+}, c_{n, m}^{-}\right)$and five eigenvalues remain at zero as $\epsilon \neq 0$. The total number of negative eigenvalues is reduced by one symmetry constraint ${ }^{4}$, such that eight negative eigenvalues in a constrained subspace match four pairs of imaginary eigenvalues with negative Krein signature. The only pair of purely imaginary eigenvalues with positive Krein signature is the pair $\lambda_{5,6}$ that is related to the two small positive eigenvalues in the subspace for components $\left(c_{n, m}^{+}, c_{n, m}^{-}\right)$.

\subsection{Eigenvalues of the $(1,-1)$ vortex cross}

Since the stability problem (4.1) has no block-diagonalization for the $(1,-1)$ vortex cross, the results of the second-order LS reductions give only two pairs of purely imaginary eigenvalues $\lambda_{1,2}$ and $\lambda_{3,4}$. We shall study the eigenvalues of the fourth-order LS reduction by using the symbolic computation package based on Wolfram's Mathematica. In order to prepare for symbolic computations, we note that the eigenvalues of $\mathcal{H}(\epsilon)$ in the case $(1,-1)$ are exactly the same as eigenvalues of $\mathcal{H}(\epsilon)$ in the case $(1,1)$, due to the equivalent transformation of the vector $\boldsymbol{\varphi}$ in the eigenvalue problem $\mathcal{H}(\epsilon) \boldsymbol{\varphi}=\gamma \boldsymbol{\varphi}$ :

$$
(1,-1):\left(\begin{array}{c}
a_{n, m} \\
b_{n, m} \\
c_{n, m}^{+} \\
c_{n, m}^{-}
\end{array}\right)=\left(\begin{array}{cccc}
\cos \delta & 0 & 0 & \sin \delta \\
0 & \cos \delta & \sin \delta & 0 \\
0 & -\sin \delta & \cos \delta & 0 \\
-\sin \delta & 0 & 0 & \cos \delta
\end{array}\right)\left(\begin{array}{c}
\varphi_{1} \\
\varphi_{2} \\
\varphi_{3} \\
\varphi_{4}
\end{array}\right)_{n, m}
$$

As a result of this transformation, we immediately find the five-dimensional kernel of $\mathcal{H}(\epsilon)$ for $\epsilon \neq 0$, which can be spanned as follows:

$\boldsymbol{\varphi}_{n, m}=\left\{\left(\begin{array}{c}\cos \delta \Phi_{n, m} \\ -\cos \delta \bar{\Phi}_{n, m} \\ -\sin \delta \bar{\Phi}_{n, m} \\ \sin \delta \Phi_{n, m}\end{array}\right),\left(\begin{array}{c}-\sin \delta \Phi_{n, m} \\ 0 \\ 0 \\ \cos \delta \Phi_{n, m}\end{array}\right),\left(\begin{array}{c}0 \\ -\sin \delta \Phi_{n, m} \\ \cos \delta \Phi_{n, m} \\ 0\end{array}\right),\left(\begin{array}{c}-\sin \delta \bar{\Phi}_{n, m} \\ 0 \\ 0 \\ \cos \delta \bar{\Phi}_{n, m}\end{array}\right),\left(\begin{array}{c}0 \\ -\sin \delta \bar{\Phi}_{n, m} \\ \cos \delta \bar{\Phi}_{n, m} \\ 0\end{array}\right)\right.$,

for $(n, m) \in \mathbb{Z}^{2}$. Algebraic multiplicity of zero eigenvalue for $\epsilon \neq 0$ is defined by the solution of the inhomogeneous equation $\mathcal{H}(\epsilon) \tilde{\varphi}=2 i \sigma \varphi$, which is equivalent to the projection equations

$$
\sum_{(n, m) \in \mathbb{Z}^{2}}\left\langle\boldsymbol{\varphi}_{j}, \sigma \varphi\right\rangle=0, \quad j=1,2,3,4,5
$$

\footnotetext{
${ }^{4}$ The Hessian matrix related to two gauge symmetry constraints has a zero eigenvalue for $\beta=1$, while only positive eigenvalues are counted in a reduction of the negative index of $\mathcal{H}(\epsilon)$.
} 
where $\varphi$ is spanned by five eigenvectors $\varphi_{j}$ in the decomposition (4.7). Solving this system of linear equations, we have found under the non-degeneracy condition (5.1) that there is a one-parameter solution of the inhomogeneous system for $\delta \neq \frac{\pi}{4}$ and a three-parameter solution for $\delta=\frac{\pi}{4}$. Thus, the zero eigenvalue has algebraic multiplicity six for $\delta \neq \frac{\pi}{4}$ and eight for $\delta=\frac{\pi}{4}$.

In the limit $\epsilon=0$, when $\mathcal{H}^{(0)}=\mathcal{H}(0)$, we construct explicitly three sets of linearly independent eigenvectors of $\mathcal{H}^{(0)}$ :

$$
\mathbf{e}_{j}=i\left(\begin{array}{c}
\cos \delta e^{i \theta_{j}} \\
-\cos \delta e^{-i \theta_{j}} \\
\sin \delta e^{-i \theta_{j}} \\
-\sin \delta e^{i \theta_{j}}
\end{array}\right), \quad \mathbf{f}_{j}^{+}=i\left(\begin{array}{c}
\cos \delta e^{i \theta_{j}} \\
-\cos \delta e^{-i \theta_{j}} \\
-\sin \delta e^{-i \theta_{j}} \\
\sin \delta e^{i \theta_{j}}
\end{array}\right), \quad \mathbf{f}_{j}^{-}=\left(\begin{array}{c}
\sin \delta e^{i \theta_{j}} \\
\sin \delta e^{-i \theta_{j}} \\
-\cos \delta e^{-i \theta_{j}} \\
-\cos \delta e^{i \theta_{j}}
\end{array}\right)
$$

Only the set of eigenvectors $\mathbf{e}_{j}$ generates the set of generalized eigenvectors of the problem $\mathcal{H}^{(0)} \hat{\mathbf{e}}_{j}=2 i \sigma \mathbf{e}_{j}$, where

$$
\hat{\mathbf{e}}_{j}=\left(\begin{array}{c}
\cos \delta e^{i \theta_{j}} \\
\cos \delta e^{-i \theta_{j}} \\
\sin \delta e^{-i \theta_{j}} \\
\sin \delta e^{i \theta_{j}}
\end{array}\right)
$$

Thus, the zero eigenvalue of $\mathcal{H}^{(0)}$ has algebraic multiplicity sixteen and geometric multiplicity twelve. Two pairs of purely imaginary eigenvalues of negative Krein signatures bifurcate at the second-order LS reductions as

$$
\lambda_{1,2}, \lambda_{3,4}= \pm 2 i \epsilon+\mathrm{O}\left(\epsilon^{2}\right) .
$$

In order to study bifurcations of non-zero eigenvalues at the fourth-order LS reductions, we consider the extended perturbation series (3.8) for $\varphi$ and $\lambda$ with $\lambda_{1}=0$ and

$$
\boldsymbol{\varphi}^{(0)}=\sum_{j=1}^{4} c_{j} \mathbf{e}_{j}+\sum_{j=1}^{4} d_{j}^{+} \mathbf{f}_{j}^{+}+\sum_{j=1}^{4} d_{j}^{-} \mathbf{f}_{j}^{-} .
$$

Performing computations symbolically, we have twelve homogeneous equations at the order of $\mathrm{O}\left(\epsilon^{2}\right)$ for twelve variables $\left(c_{j}, d_{j}^{+}, d_{j}^{-}\right), j=1,2,3,4$, which can be converted and simplified to the following determinant equation:

$$
\gamma_{2}^{2}+4(1+4 \cos 4 \delta) \gamma_{2}+36=0
$$

where $\gamma_{2}=\frac{1}{2} \lambda_{2}^{2}$. By using the inverse relation $\lambda_{2}= \pm \sqrt{2 \gamma_{2}}$ and finding the roots for $\gamma_{2}$ explicitly, we obtain four small pairs of eigenvalues with asymptotic approximations:

$$
\begin{aligned}
& \lambda_{5,6}= \pm 2 i \epsilon^{2} \sqrt{1+4 \cos 4 \delta-\sqrt{8(\cos 4 \delta+\cos 8 \delta)}}+\mathrm{O}\left(\epsilon^{3}\right), \\
& \lambda_{7,8}= \pm 2 i \epsilon^{2} \sqrt{1+4 \cos 4 \delta+\sqrt{8(\cos 4 \delta+\cos 8 \delta)}}+\mathrm{O}\left(\epsilon^{3}\right) .
\end{aligned}
$$


When $\delta=0$ or $\delta=\frac{\pi}{2}$, we obtain the same pairs of purely imaginary eigenvalues as in the case $(1,1)$ (see Proposition [5.1). When $\delta=\frac{\pi}{4}$, we obtain two degenerate pairs of real eigenvalues

$$
\lambda_{5,6}, \lambda_{7,8}= \pm 2 \sqrt{3} \epsilon^{2}+\mathrm{O}\left(\epsilon^{3}\right) .
$$

The instability domain is found analytically from the condition that complex-valued roots for $\gamma_{2}$ coalesce and become a double negative root. This happens when $\cos (4 \delta)+\cos (8 \delta)=0$, which is solved on the interval $\delta \in\left[0, \frac{\pi}{2}\right]$ at $\delta=\frac{\pi}{12}$ and $\delta=\frac{5 \pi}{12} 5$ Thus, the instability domain of the $(1,-1)$ vortex cross in the case $\beta=1$ is bounded by the interval $\delta \in\left(\frac{\pi}{12}, \frac{5 \pi}{12}\right)$.

In order to capture the remaining pair of non-zero eigenvalues $\lambda_{9,10}$, we shall reorder the perturbation series expansions and to move the last two sums in the decomposition (5.6) to the order of $\mathrm{O}\left(\epsilon^{2}\right)$, while the coefficients of the vector $\mathbf{c}=\left(c_{1}, c_{2}, c_{3}, c_{4}\right)^{T}$ should be projected to the vector $\mathbf{p}_{2}=(-1,1,-1,1)$ of the kernel of $\mathcal{M}_{2}$, such that $\mathbf{c}=x_{1} \mathbf{p}_{2}$. Performing computations symbolically, we have twelve homogeneous equations at the order of $\mathrm{O}\left(\epsilon^{4}\right)$ for eight variables in the vectors $\mathbf{d}^{+}$and $\mathbf{d}_{j}^{-}$and the coordinate $x_{1}$. The homogeneous system is satisfied with the choice $\mathbf{d}^{+}=\mathbf{0}$ and $\mathbf{d}^{-}=x_{2} \mathbf{p}_{2}$, where $x_{2}$ is another coordinate. The coordinates $\left(x_{1}, x_{2}\right)$ solve a homogeneous system with the determinant equation $\lambda_{2}^{2}=-16 \cos ^{2}(2 \delta)$. Therefore, a small pair of purely imaginary eigenvalues of negative Krein signatures has the asymptotic approximation:

$$
\lambda_{9,10}= \pm 4 i \epsilon^{2} \cos (2 \delta)+\mathrm{O}\left(\epsilon^{3}\right)
$$

When $\delta=0$ and $\delta=\frac{\pi}{2}$, the pair $\lambda_{9,10}$ matches to that in the case $(1,1)$ (see Proposition 5.1). When $\delta=\frac{\pi}{4}$, the pair remains at the origin as it follows from the study of algebraic multiplicity of zero eigenvalue. According to the count of negative eigenvalues, the total number of negative eigenvalues of $\mathcal{H}(\epsilon)$ for small $\epsilon$ reduced by one symmetry constraint is eight. These eigenvalues match two pairs of imaginary eigenvalues $\lambda_{1,2}$ and $\lambda_{3,4}$ and two real positive eigenvalues $\lambda_{5,6}$ and $\lambda_{7,8}$.

Asymptotic and numerical approximations of small eigenvalues $\lambda$ for small values of $\epsilon$ for $\omega=\beta=1$ and $\delta=\frac{\pi}{4}$ are shown on Figure [5. The left plot corresponds to the vortex pair $(1,1)$, while the right plot corresponds to the vortex pair $(1,-1)$. We can see that the $(1,1)$ vortex cross is linearly stable in the anti-continuum limit, according to the results of Proposition 5.1. On the other hand, the $(1,-1)$ vortex cross become unstable because of the a double pairs of real eigenvalues $\lambda_{5,6}=\lambda_{7,8}$. The other double pair of purely imaginary eigenvalues remains double for all $\epsilon>0$, such that $\lambda_{1,2}=\lambda_{3,4}$. Therefore, the stability changes

\footnotetext{
${ }^{5}$ Another solution exists at $\delta=\frac{\pi}{4}$ but it corresponds to the case when complex-valued roots coalesce and become a double positive root for $\gamma_{2}$.

${ }^{6}$ Eigenvalues $\lambda_{5,6}$ and $\lambda_{7,8}$ are real only in the case $\delta=\frac{\pi}{4}$. For other values of $\delta$, these eigenvalues are either complex-valued or purely imaginary. The count is not affected, since two real eigenvalues are equivalent to four complex eigenvalues which may coalesce due to the inverse Hamilton-Hopf bifurcation to two pairs of purely imaginary eigenvalues with positive and negative Krein signatures.
} 

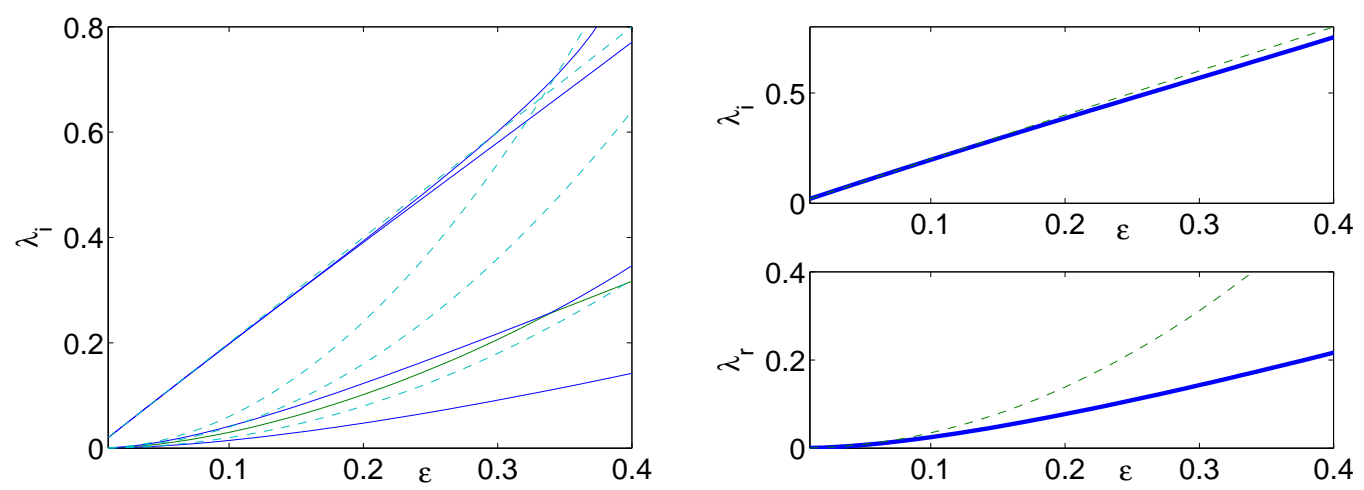

Figure 5: Eigenvalues of the vector vortex cross with $\omega=\beta=1$ and $\delta=\frac{\pi}{4}$ versus $\epsilon$. Left: $(1,1)$. Right: $(1,-1)$. The solid lines show the full numerical results, while the dashed lines show the asymptotic approximations. Bolded curves show double eigenvalues.

drastically in the case of the discrete Manakov system (that is the coupled DNLS system for $\beta=1)$ : the $(1,1)$ vortex cross is stable near the anti-continuum limit while the $(1,-1)$ vortex cross is linearly unstable.

\section{Conclusions}

We have examined analytically and numerically the existence and stability of vortex cross configurations in the single-component and two-component DNLS equations. We have used the Lyapunov-Schmidt theory, to obtain the bifurcation functions and the solvability conditions that allow persistence of such configurations near the anti-continuum limit. Additionally, the theory gives analytical expressions for eigenvalues of the linearized stability problem as functions of the system parameters (namely, the coupling between adjacent lattice nodes $\epsilon \geq 0$ and the coupling between the two components $\beta \geq 0$ ).

One of the interesting recent experimental developments in the setting of BECs concerns the experimental and theoretical studies of spin-1 (or 3-component) states 42, 43. This feature, along with similar possibilities that could be realized in optical settings, render desirable a general theory for interactions of multiple components. Such studies are currently in progress. 


\section{A Continuation of the single-component vortex cross}

We apply the algorithm of Lyapunov-Schmidt (LS) reductions (see 9] for details) and compute the first few terms of the perturbation series expansions:

$$
\Phi_{n, m}(\epsilon)=\sum_{k=0}^{\infty} \epsilon^{k} \Phi_{n, m}^{(k)}
$$

The zero-order solution $\Phi_{n, m}^{(0)}$ is given by (3.2). The first-order correction is obtained in the explicit form:

$$
\Phi_{n, m}^{(1)}=\left\{\begin{array}{l}
0, \quad(n, m) \in S^{(0)} \\
\sum_{l}^{(1)} e^{i \theta_{l},} \quad(n, m) \in S^{(1)} \\
0, \quad(n, m) \notin S^{(0)} \cup S^{(1)}
\end{array}\right.
$$

where $S^{(1)}$ is the set of adjacent nodes to the set $S^{(0)}$ and $\sum_{l}^{(1)} e^{i \theta_{l}}$ is a schematic notation for the following solution:

$$
\sum_{l}^{(1)} e^{i \theta_{l}}=\left\{\begin{array}{l}
e^{i \theta_{1}}+e^{i \theta_{2}}+e^{i \theta_{3}}+e^{i \theta_{4}}, \quad(n, m)=(0,0) \\
e^{i \theta_{j}}+e^{\theta_{j+1}}, \quad(n, m)=\{(-1,-1) ;(1,-1) ;(1,1) ;(-1,1)\} \\
e^{i \theta_{j}}, \quad(n, m)=\{(-2,0) ;(0,-2) ;(2,0) ;(0,2)\}
\end{array}\right.
$$

The index $j$ enumerates nodes in the set $S^{(0)}$ that are adjacent to the nodes in the set $S^{(1)}$ listed in the figured brackets of (A.3). No non-trivial bifurcation equations arise at the firstorder reductions, i.e. the first-order correction to the bifurcation function $\mathbf{g}^{(1)}(\boldsymbol{\theta})$ is zero, where $\boldsymbol{\theta}=\left(\theta_{1}, \theta_{2}, \theta_{3}, \theta_{4}\right)$ and notations of [9] are used. The second-order correction is found in the form:

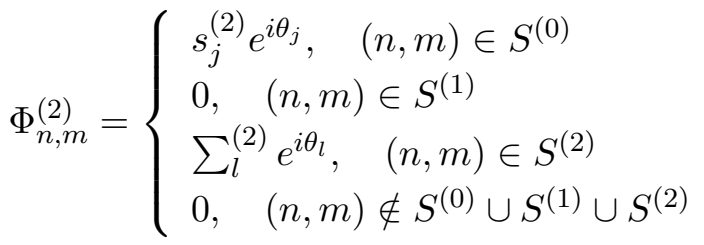

where

$$
-2 s_{j}^{(2)}=4+2 \cos \left(\theta_{j+1}-\theta_{j}\right)+2 \cos \left(\theta_{j-1}-\theta_{j}\right)+\cos \left(\theta_{j+2}-\theta_{j}\right) .
$$

The set $S^{(2)}$ contains outward adjacent nodes to the set $S^{(1)} \backslash\{(0,0)\}$ and $\sum_{l}^{(2)} e^{i \theta_{l}}$ is a schematic notation for the following solution:

$$
\sum_{l}^{(2)} e^{i \theta_{l}}=\left\{\begin{array}{l}
2 e^{i \theta_{j}}+e^{i \theta_{j+1}}, \quad(n, m)=\{(-2,-1) ;(1,-2) ;(2,1) ;(-1,2)\} \\
e^{i \theta_{j}}+2 e^{\theta_{j+1}}, \quad(n, m)=\{(-1,-2) ;(2,-1) ;(1,2) ;(-2,1)\} \\
e^{i \theta_{j}}, \quad(n, m)=\{(-3,0) ;(0,-3) ;(3,0) ;(0,3)\}
\end{array}\right.
$$

The second-order corrections to the bifurcation function take the form:

$$
g_{j}^{(2)}=2 \sin \left(\theta_{j}-\theta_{j+1}\right)+2 \sin \left(\theta_{j}-\theta_{j-1}\right)+\sin \left(\theta_{j}-\theta_{j+2}\right), \quad j=1,2,3,4 .
$$


The bifurcation equations $\mathbf{g}^{(2)}(\boldsymbol{\theta})=\mathbf{0}$ are satisfied with the one-parameter family of asymmetric vortices:

$$
\theta_{1}=0, \quad \theta_{2}=\theta, \quad \theta_{3}=\pi, \quad \theta_{4}=\pi+\theta
$$

where $\theta \in(0, \pi)$. When $\theta=\frac{\pi}{2}$, the family (A.7) reduces to the vortex cross configuration (2.12). The Jacobian matrix $\mathcal{M}_{2}$ of the second-order bifurcation function $\mathbf{g}^{(2)}(\boldsymbol{\theta})$ is obtained by differentiation of $\mathbf{g}^{(2)}$ in $\boldsymbol{\theta}$. At the family of asymmetric vortices (A.7), the Jacobian matrix $\mathcal{M}_{2}$ takes the form:

$$
\mathcal{M}_{2}=\left(\begin{array}{cccc}
-1 & -2 \cos \theta & 1 & 2 \cos \theta \\
-2 \cos \theta & -1 & 2 \cos \theta & 1 \\
1 & 2 \cos \theta & -1 & -2 \cos \theta \\
2 \cos \theta & 1 & -2 \cos \theta & -1
\end{array}\right) .
$$

It has two zero eigenvalues and two non-zero eigenvalues $-2 \pm 4 \cos \theta$. In the case of the vortex $\operatorname{cross}\left(\theta=\frac{\pi}{2}\right)$, it has two zero eigenvalues and two negative eigenvalues -2 . The third-order correction satisfies the inhomogeneous equation,

$$
\left(1-2\left|\Phi_{n, m}^{(0)}\right|^{2}\right) \Phi_{n, m}^{(3)}-\Phi_{n, m}^{(0) 2} \bar{\Phi}_{n, m}^{(3)}=\Phi_{n+1, m}^{(2)}+\Phi_{n-1, m}^{(2)}+\Phi_{n, m+1}^{(2)}+\Phi_{n, m-1}^{(2)}+\left|\Phi_{n, m}^{(1)}\right|^{2} \Phi_{n, m}^{(1)}
$$

where we have shorten nonlinear terms, since $\Phi_{n, m}^{(0)} \Phi_{n, m}^{(1)}=\Phi_{n, m}^{(1)} \Phi_{n, m}^{(2)}=0$ for all $(n, m) \in \mathbb{Z}^{2}$. The third-order correction is found in the form:

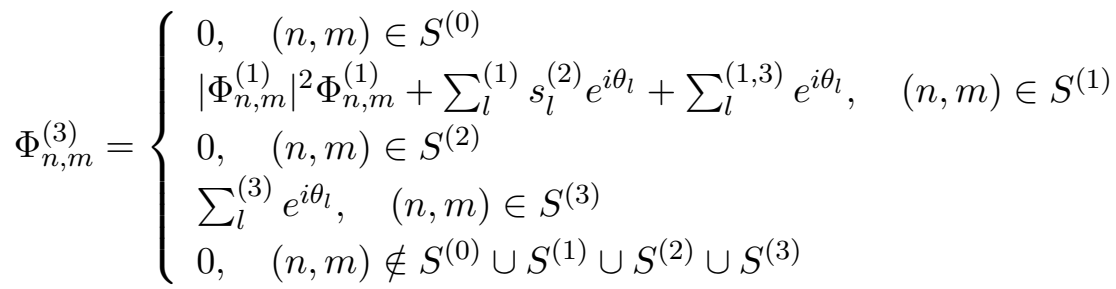

where the sum $\sum_{l}^{(1)} s_{l}^{(2)} e^{i \theta_{l}}$ is defined similarly to the sum (A.3), the sum $\sum_{l}^{(3)} e^{i \theta_{l}}$ is not used for further computations, and the sum $\sum_{l}^{(1,3)} e^{i \theta_{l}}$ is defined as follows:

$$
\sum_{l}^{(1,3)} e^{i \theta_{l}}=\left\{\begin{array}{l}
3 e^{i \theta_{j}}+3 e^{\theta_{j+1}}, \quad(n, m)=\{(-1,-1) ;(1,-1) ;(1,1) ;(-1,1)\} \\
5 e^{i \theta_{j}}+e^{i \theta_{j+1}}+e^{i \theta_{j-1}}, \quad(n, m)=\{(-2,0) ;(0,-2) ;(2,0) ;(0,2)\}
\end{array}\right.
$$

No non-trivial bifurcation equations arise at the third-order reductions, i.e. $\mathbf{g}^{(3)}(\boldsymbol{\theta})=\mathbf{0}$. The fourth-order correction satisfies the inhomogeneous equation,

$\left(1-2\left|\Phi_{n, m}^{(0)}\right|^{2}\right) \Phi_{n, m}^{(4)}-\Phi_{n, m}^{(0) 2} \bar{\Phi}_{n, m}^{(4)}=2\left|\Phi_{n, m}^{(2)}\right|^{2} \Phi_{n, m}^{(0)}+\Phi_{n, m}^{(2) 2} \bar{\Phi}_{n, m}^{(0)}+\Phi_{n+1, m}^{(3)}+\Phi_{n-1, m}^{(3)}+\Phi_{n, m+1}^{(3)}+\Phi_{n, m-1}^{(3)}$.

Solving the inhomogeneous equation for the third-order corrections, we obtain the bifurcation equations at the fourth order of LS reductions in the form:

$g_{j}^{(4)}=\left(4+2 \cos \left(\theta_{j+2}-\theta_{j+1}\right)+2 \cos \left(\theta_{j}-\theta_{j+1}\right)+\cos \left(\theta_{j-1}-\theta_{j+1}\right)\right) \sin \left(\theta_{j+1}-\theta_{j}\right)$ 


$$
\begin{array}{ll}
+ & \left(4+2 \cos \left(\theta_{j-2}-\theta_{j-1}\right)+2 \cos \left(\theta_{j}-\theta_{j-1}\right)+\cos \left(\theta_{j+1}-\theta_{j-1}\right)\right) \sin \left(\theta_{j-1}-\theta_{j}\right) \\
+ & \frac{1}{2}\left(4+2 \cos \left(\theta_{j-1}-\theta_{j-2}\right)+2 \cos \left(\theta_{j+1}-\theta_{j+2}\right)+\cos \left(\theta_{j}-\theta_{j+2}\right)\right) \sin \left(\theta_{j+2}-\theta_{j}\right) \\
+ & \frac{1}{2}\left(4+2 \cos \left(\theta_{j+1}-\theta_{j+2}\right)+2 \cos \left(\theta_{j-1}-\theta_{j-2}\right)+\cos \left(\theta_{j}-\theta_{j-2}\right)\right) \sin \left(\theta_{j-2}-\theta_{j}\right) \\
+ & 2\left(1+\cos \left(\theta_{j+1}-\theta_{j}\right)\right) \sin \left(\theta_{j}-\theta_{j+1}\right)+2\left(1+\cos \left(\theta_{j-1}-\theta_{j}\right)\right) \sin \left(\theta_{j}-\theta_{j-1}\right) \\
+ & 2\left(2+\cos \left(\theta_{2}-\theta_{1}\right)+\cos \left(\theta_{3}-\theta_{1}\right)+\cos \left(\theta_{4}-\theta_{1}\right)+\cos \left(\theta_{3}-\theta_{2}\right)+\cos \left(\theta_{4}-\theta_{2}\right)+\cos \left(\theta_{4}-\theta_{3}\right)\right) \\
& \times\left(\sin \left(\theta_{j}-\theta_{j+1}\right)+\sin \left(\theta_{j}-\theta_{j-1}\right)+\sin \left(\theta_{j}-\theta_{j+2}\right)\right) \\
+ & 4 \sin \left(\theta_{j}-\theta_{j+1}\right)+4 \sin \left(\theta_{j}-\theta_{j-1}\right)
\end{array}
$$

For the asymmetric vortex, we have

$$
g_{j}^{(4)}=(-1)^{j} 2 \sin (2 \theta), \quad j=1,2,3,4 .
$$

The Jacobian matrix $\mathcal{M}_{2}$ has two zero eigenvalues with orthogonal eigenvectors:

$$
\mathbf{p}_{1}=\left(\begin{array}{c}
1 \\
1 \\
1 \\
1
\end{array}\right), \quad \mathbf{p}_{2}=\left(\begin{array}{c}
-1 \\
1 \\
-1 \\
1
\end{array}\right)
$$

It is clear that the vector $\mathbf{g}^{(4)}=2 \sin (2 \theta) \mathbf{p}_{2}$ is not orthogonal to the eigenvector $\mathbf{p}_{2}$ of the kernel of $\mathcal{M}_{2}$, unless $\theta=\left\{0, \frac{\pi}{2}, \pi\right\}$. By Proposition 2.10 in [9], the family of asymmetric vortices (A.7) terminates at the fourth-order reduction. The exceptional cases include discrete solitons for $\theta=\{0, \pi\}$ and the vortex cross at $\theta=\frac{\pi}{2}$. In order to consider persistence of the vortex cross, we compute the Jacobian matrices $\mathcal{M}_{2}$ and $\mathcal{M}_{4}$ from the bifurcation functions $\mathbf{g}^{(2)}$ and $\mathbf{g}^{(4)}$ explicitly,

$$
\mathcal{M}_{2}=\left(\begin{array}{cccc}
-1 & 0 & 1 & 0 \\
0 & -1 & 0 & 1 \\
1 & 0 & -1 & 0 \\
0 & 1 & 0 & -1
\end{array}\right), \quad \mathcal{M}_{4}=\left(\begin{array}{cccc}
3 & 2 & -7 & 2 \\
2 & 3 & 2 & -7 \\
-7 & 2 & 3 & 2 \\
2 & -7 & 2 & 3
\end{array}\right)
$$

Since $\mathcal{M}_{4} \mathbf{p}_{1}=\mathbf{0}$ and $\mathcal{M}_{4} \mathbf{p}_{2} \neq \mathbf{0}$, the zero eigenvalue of $\mathcal{M}_{2}$ with the associated eigenvector $\mathbf{p}_{2}$ bifurcates. By Proposition 2.9 in [9], this implies that the family of the vortex cross is continued from the anti-continuum limit uniquely up to the rotational transformation $\boldsymbol{\theta} \rightarrow \boldsymbol{\theta}+\theta_{0} \mathbf{p}_{1}$ that corresponds to the gauge symmetry of the dNLS equation (3.1). Proposition 3.1] is hence proved.

Small eigenvalues of the linearized Jacobian matrix $\mathcal{H}(\epsilon)$ are defined by an extended eigenvalue problem for the Jacobian matrices $\mathcal{M}_{2}$ and $\mathcal{M}_{4}$,

$$
\left(\epsilon^{2} \mathcal{M}_{2}+\epsilon^{4} \mathcal{M}_{4}+\mathrm{O}\left(\epsilon^{6}\right)\right) \mathbf{c}=\gamma \mathbf{c}
$$


There exist four eigenvalues of the extended problem which admit the asymptotic approximations,

$$
\gamma_{1,2}=-2 \epsilon^{2}+\mathrm{O}\left(\epsilon^{4}\right), \quad \gamma_{3}=-8 \epsilon^{4}+\mathrm{O}\left(\epsilon^{6}\right), \quad \gamma_{4}=0 .
$$

The eigenvalue $\gamma_{3}$ is obtained by the perturbation theory for the zero eigenvalue of $\mathcal{M}_{2}$ associated with the eigenvector $\mathbf{p}_{2}$ (orthogonal to the eigenvector $\mathbf{p}_{1}$ ):

$$
\lim _{\epsilon \rightarrow 0} \epsilon^{-4} \gamma_{3}=\frac{\left(\mathbf{p}_{2}, \mathcal{M}_{4} \mathbf{p}_{2}\right)}{\left(\mathbf{p}_{2}, \mathbf{p}_{2}\right)}=-8
$$

\section{References}

[1] J.W. Fleischer, G. Bartal, O. Cohen, T. Schwartz, O. Manela, B. Freedman, M. Segev, H. Buljan, and N.K. Efremidis, "Spatial photonics in nonlinear waveguide arrays", Opt. Express 13, 1780 (2005).

[2] V.A. Brazhnyi and V.V. Konotop, "Theory of nonlinear matter waves in optical lattices", Mod. Phys. Lett. B 18, 627 (2004)

[3] P.G. Kevrekidis and D.J. Frantzeskakis, "Pattern forming dynamical instabilities in BoseEinstein condensates", Mod. Phys. Lett. B 18, 173 (2004)

[4] O. Morsch and E. Arimondo, in Dynamics and Thermodynamics of Systems with LongRange Interactions, T. Dauxois, S. Ruffo, E. Arimondo and M. Wilkens (Eds.), Springer (Berlin 2002), pp. 312-331.

[5] P.G. Kevrekidis, K.Ø. Rasmussen and A.R. Bishop, "The discrete nonlinear Schrödinger equation: a survey of recent results", Int. J. Mod. Phys. B 15, 2833 (2001)

[6] G.L. Alfimov, P.G. Kevrekidis, V.V. Konotop and M. Salerno, "Wannier functions analysis of the nonlinear Schrödinger equation with a periodic potential", Phys. Rev. E 66, $046608(2002)$

[7] Yu.S. Kivshar and M. Peyrard, Phys. Rev. A 46, 3198 (1992).

[8] D.E. Pelinovsky, P.G. Kevrekidis, and D.J. Frantzeskakis, "Stability of discrete solitons in nonlinear Schrödinger lattices", nlin.PS/0410005, Physica D, in press (2005).

[9] D.E. Pelinovsky, P.G. Kevrekidis, and D.J. Frantzeskakis, "Persistence and stability of discrete vortices in nonlinear Schrödinger lattices", nlin.PS/0411016, Physica D, in press (2005).

[10] J.W. Fleischer, T. Carmon, M. Segev, N.K. Efremidis and D.N. Christodoulides, "Observation of discrete solitons in optically induced real time waveguide arrays", Phys. Rev. Lett. 90023902 (2003) 
[11] H. Martin, E.D. Eugenieva, Z. Chen and D.N. Christodoulides, "Discrete solitons and soliton-induced dislocations in partially coherent photonic lattices", Phys. Rev. Lett. 92 123902 (2004)

[12] J. Yang, I. Makasyuk, A. Bezryadina and Z. Chen, "Dipole solitons in optically-induced two-dimensional photonic lattices", Opt. Lett. 29, 1662 (2004)

[13] Z. Chen, H. Martin, E.D. Eugenieva, J. Xu and A. Bezryadina, "Anisotropic enhancement of discrete diffraction and formation of two-dimensional discrete-soliton trains", Phys. Rev. Lett. 92143902 (2004)

[14] J. Yang, I. Makasyuk, P.G. Kevrekidis, H. Martin, B.A. Malomed, D.J. Frantzeskakis, and Z. Chen, "Necklace-like solitons in optically induced photonic lattices", Phys. Rev. Lett. 94, 113902 (2005)

[15] Z. Chen, I. Makasyuk, A. Bezryadina and J. Yang, "Observation of two-dimensional lattice vector solitons", Opt. Lett. 291656 (2004)

[16] D.N. Neshev, T.J. Alexander, E.A. Ostrovskaya, Yu.S. Kivshar, H. Martin, I. Makasyuk and Z. Chen, "Observation of discrete vortex solitons in optically induced photonic lattices", Phys. Rev. Lett. 92, 123903 (2004)

[17] J.W. Fleischer, G. Bartal, O. Cohen, O. Manela, M. Segev, J. Hudock and D.N. Christodoulides, "Observation of vortex-ring "discrete" solitons in 2D photonic lattices", Phys. Rev. Lett. 92 (2004) 123904

[18] B.A. Malomed and P.G. Kevrekidis, "Discrete vortex solitons", Phys. Rev. E 64, 026601 (2001)

[19] J. Yang and Z. Musslimani, "Fundamental and vortex solitons in a two-dimensional optical lattice", Opt. Lett. 23, 2094 (2003)

[20] P.G. Kevrekidis, B.A. Malomed, Z. Chen and D.J. Frantzeskakis, "Stable higher-order vortices and quasi-vortices in the discrete nonlinear Schrödinger equation", Phys. Rev. E 70,056612 (2004).

[21] S. Burger, K. Bongs, S. Dettmer, W. Ertmer, K. Sengstock, A. Sanpera, G.V. Shlyapnikov and M. Lewenstein, "Dark solitons in Bose-Einstein condensates", Phys. Rev. Lett. 83, 5198 (1999)

[22] J. Denschlag, J.E. Simsarian, D.L. Feder, C.W. Clark, L.A. Collins, J. Cubizolles, L. Deng, E.W. Hagley, K. Helmerson, W.P. Reinhardt, S.L. Rolston, B.I. Schneider and W.D. Phillips, "Generating solitons by phase engineering of a Bose-Einstein condensate", Science 287, 97 (2000) 
[23] B.P. Anderson, P.C. Haljan, C.A. Regal, D.L. Feder, L.A. Collins, C.W. Clark and E.A. Cornell, "Watching dark solitons decay into vortex rings in a Bose-Einstein condensate", Phys. Rev. Lett. 86, 2926 (2001)

[24] K. E. Strecker, G.B. Partridge, A.G. Truscott and R.G. Hulet, "Formation and propagation of matter-wave soliton trains", Nature 417, 150 (2002)

[25] L. Khaykovich, F. Schreck, G. Ferrari, T. Bourdel, J. Cubizolles, L.D. Carr, Y. Castin and C. Salomon, "Formation of a matter-wave bright soliton", Science 296, 1290 (2002)

[26] B. Eiermann, Th. Anker, M. Albiez, M. Taglieber, P. Treutlein, K.-P. Marzlin and M.K. Oberthaler, "Bright Bose-Einstein gap solitons of atoms with repulsive interaction", Phys. Rev. Lett. 92, 230401 (2004).

[27] M. Greiner, I. Bloch, O. Mandel, T.W. Hänsch and T. Esslinger, "Bose-Einstein Condensates in 1D and 2D optical lattices", Appl. Phys. B 47, 769 (2001) and "Exploring phase coherence in a 2D lattice of Bose-Einstein condensates", Phys. Rev. Lett. 87, 160405 (2001).

[28] J. Meier, J. Hudock, D. Christodoulides, G. Stegeman, Y. Silberberg, R. Morandotte and J.S. Aitchison, "Discrete vector solitons in Kerr nonlinear waveguide arrays", Phys. Rev. Lett. 91, 143907 (2003)

[29] J. Meier, J. Hudock, D.N. Christodoulides, G.I. Stegeman, H.Y. Yang, G. Salamo, R. Morandotti, J.S. Aitchison and Y. Silberberg, "Excitation of strongly confined scalar and vector self-trapped beams in one-dimensional arrays of Kerr-nonlinear channel waveguides", J. Opt. Soc. Am. B 22, 1432 (2005).

[30] C. J. Myatt, E.A. Burt, R.W. Ghrist, E.A. Cornell and C.E. Wieman, "Production of two overlapping Bose-Einstein condensates by sympathetic cooling", Phys. Rev. Lett. 78, 586 (1997)

[31] D.S. Hall, M.R. Matthews, J.R. Ensher, C.E. Wieman and E.A. Cornell, "Dynamics of component separation in a binary mixture of Bose-Einstein condensates", Phys. Rev. Lett. 81, 1539 (1998)

[32] D.M. Stamper-Kurn, M.R. Andrews, A.P. Chikkatur, S. Inouye, H.-J. Miesner, J. Stenger and W. Ketterle, "Optical confinement of a Bose-Einstein condensate", Phys. Rev. Lett. 80, 2027 (1998)

[33] G. Modugno, G. Ferrari, G. Roati, R.J. Brecha, A. Simoni and M. Inguscio, "BoseEinstein condensation of potassium atoms by sympathetic cooling", Science 294, 1320 (2001) 
[34] M. Mudrich, S. Kraft, K. Singer, R. Grimm, A. Mosk and M. Weidemüller, "Sympathetic cooling with two atomic species in an optical trap", Phys. Rev. Lett. 88, 253001 (2002).

[35] F. Lederer, S. Darmanyan and A. Kobyakov in Spatial Optical Solitons, edited by S. Trillo and W.E. Torruellas (Springer-Verlag, New York, 2001)

[36] M.J. Ablowitz and Z.H. Musslimani, "Discrete vector spatial solitons in a nonlinear waveguide array", Phys. Rev. E 65, 056618 (2002)

[37] J. Hudock, P. G. Kevrekidis, B. A. Malomed, and D. N. Christodoulides, "Discrete vector solitons in two-dimensional nonlinear waveguide arrays: solutions, stability and dynamics", Phys. Rev. E 67, 056618 (2003)

[38] R.A. Vicencio, M.I. Molina and Yu.S. Kivshar, "All-optical switching and amplification of discrete vector solitons in nonlinear cubic birefringent waveguide arrays", Opt. Lett. 29, 2905 (2004) and "Polarization instability, steering and switching of discrete vector solitons", Phys. Rev. E 71, 056613 (2005)

[39] A.S. Desyatnikov, D. Michalache, D. Mazilu, B.A. Malomed, C. Denz and F. Lederer, "Two-dimensional solitons with hidden and explicit vorticity in bimodal cubic-quintic media", Phys. Rev. E 71, 026615 (2005)

[40] F. Ye, J. Wang, L. Dong and Y.-P. Li, "Suppression of modulational instability of ring vector solitons", Opt. Comm. 230, 219 (2004)

[41] D.E. Pelinovsky and Yu.S. Kivshar, "Stability criterion for multicomponent solitary waves", Phys. Rev. E 62, 8668-8676 (2000).

[42] J.M. Higbie, L.E. Sadler, S. Inouye, A.P. Chikkatur, S.R. Leslie, K.L. Moore, V. Savalli and D.M. Stamper-Kurn, "Direct nondestructive imaging of magnetization in a spin-1 Bose-Einstein gas", Phys. Rev. Lett. 95, 050401

[43] H. Saito and M. Ueda, "Spontaneous magnetization and structure formation in a spin-1 ferromagnetic Bose-Einstein condensate", cond-mat/0504398 